\title{
Safety of Modified Ultra-Rush Venom Immunotherapy in Children
}

\section{Steiß JO*, Lindemann H and Zimmer KP}

Department of Pediatrics, University Children's Hospital, Germany

\begin{abstract}
Background: As many as $5 \%$ of the population in Central Europe suffer from insect venom allergy. The protective effect of conventional specific immunotherapy is quite convincing, as it is associated with a success rate up to $95 \%$. We report our experience concerning ultra-rush dose titration in children and adolescents.

Objective: The aim of this study was to examine the safety and tolerability of a shortened insect venom immunotherapy in children.

Patients and methods: A modified version of the ultra-rush procedure was initiated for 38 bee venom and 54 wasp venom allergies in 90 patients (56 boys and 34 girls) aged 4 to 17 years. Consolidation therapy consisted of $100 \mu \mathrm{g}$ boost injections administered after seven and 21 days while maintenance therapy was administered every four to six weeks.

Results: All patients developed local reactions under VIT, of whom 20 had extensive reddening $(>5 \mathrm{~cm}-20 \mathrm{~cm})$, 15 patients developed significant wheals $(5 \mathrm{~cm}-15 \mathrm{~cm})$, and two patients with a bee venom allergy had systemic reactions which could be controlled well. Maintenance therapy was tolerated well by all patients.

Conclusion: Ultra-rush titration is safe, tolerable and effective in children and adolescents, in addition to being associated with greater compliance and shorter hospital stays when compared to conventional therapy regimens. No serious side effects were registered. All patients could be discharged from the hospital at the latest after 48 hours.
\end{abstract}

Keywords: Insect venom immunotherapy; Hymenoptera venom allergy; Insect sting; Ultra-rush immunotherapy; Safety

\section{Introduction}

As many as $5 \%$ of the population in Central Europe suffer from insect venom allergy. The protective effect of conventional specific immunotherapy for insect venom allergy (VIT) is quite convincing, as it is associated with a success rate of $95 \%$. VIT is recommended for patients with systemic anaphylactic sting reactions and evidence of IgEmediated sensitization $[1,2]$. Specific immunotherapy still is the sole causal therapy for Hymenoptera venom allergy; its safety and efficacy are accepted throughout the world. A number of treatment regimens have been published in the last few years for the preliminary phase of specific immunotherapy. These treatment regimens vary in terms of the duration of therapy, number of injections, and the administered dose of insect venom, as well as the frequency of systemic side effects during treatment [2-5].

A modified time-saving ultra-rush procedure is being used since 2002 at the University Clinic of Giessen to initiate desensitization in children and adolescents with bee or wasp venom allergy. As very few studies have been performed concerning the tolerability of the treatment especially in children, the safety and appropriateness of the ultra-rush procedure are still intensively discussed.

The aim of the present study was to investigate the tolerance and safety of this therapy option in children, and to identify potential risk factors for systemic side effects.

\section{Material and Methods}

Medical records of 90 children treated with a modified ultra-rush protocol between 2002 and 2011 were analyzed retrospectively. All patients were monitored in the hospital setting with full emergency resuscitation equipment readily available. The patients received an intravenous access; heart rate, blood pressure and ECG were monitored.
VIT was recommended when the patient had a history of an immediate systemic reaction after a sting. Detection of specific IgE antibodies to the venom and/or skin tests were performed according to the European Academy of Allergy and Immunology [6]

We selected the grading scheme proposed by Mueller HL (Table 1) [7]. The interval between the last allergic reaction and the initiation of insect VIT was 4-12 months. From 2002 to 2004, ultra-rush VIT was administered to 19 patients using a lyophilized bee venom or vespula species preparation obtained from ALK SQ, Scherax GmbH, Hamburg, and Germany. Subsequently Venomil (Bencard Company, Munich, Germany) was administered subcutaneously to 71 patients. Ultrarush titration of Venomil for this indication has been approved by the Federal Institute of Vaccines and Biomedical Drugs (Paul-Ehrlich Institute). Allergen-specific IgE levels were measured in the patients

\begin{tabular}{|l|l|}
\hline Grade 0 & Strong local reaction, diameter larger than $10 \mathrm{~cm}$, longer than $24 \mathrm{~h}$ \\
\hline Grade I & Generalized urticaria, pruritus, nausea \\
\hline Grade II & Vomiting, angioedema, dizziness, sensation of tightness \\
\hline Grade III & Dyspnea, stridor, wheezing, fear of dying, weakness, drowsiness \\
\hline Grade IV & $\begin{array}{l}\text { Drop in blood pressure, cyanosis, respiratory arrest, circulatory } \\
\text { arrest }\end{array}$ \\
\hline
\end{tabular}

Table 1: Severity of allergic reactions based on Müller et al. [7].

*Corresponding author: Jens-Oliver Steiß, Department of Pediatrics, University Children's Hospital, Division of Pediatric Pulmology and Allergy, Feulgenstraße 12 D-35385 Giessen, Germany, Tel: +49(0)-641-985-57621; Fax: +49(0)-641-98557628; E-mail: Jens-Oliver.Steiss@paediat.med.uni-giessen.de

Received April 05, 2013; Accepted April 28, 2013; Published April 30, 2013

Citation: Steiß JO, Lindemann H, Zimmer KP (2013) Safety of Modified UltraRush Venom Immunotherapy in Children. J Aller Ther 4: 134. doi:10.4172/21556121.1000134

Copyright: ( 2013 Steiß JO, et al. This is an open-access article distributed under the terms of the Creative Commons Attribution License, which permits unrestricted use, distribution, and reproduction in any medium, provided the original author and source are credited. 


\begin{tabular}{|l|c|c|}
\hline \multirow{2}{*}{ Day 1 } & Time (hours) & Insect venom $(\boldsymbol{\mu g})$ \\
\cline { 2 - 3 } & 0 & 0.01 \\
\cline { 2 - 3 } & 0.5 & 0.10 \\
\cline { 2 - 3 } & 1.0 & 1.00 \\
\cline { 2 - 3 } & 1.5 & 10.00 \\
\hline \multirow{2}{*}{ Day 2 } & 2.0 & 20.00 \\
\hline Maintenance therapy (100 $\mu \mathrm{gg})$ was administered after preliminary therapy as \\
follows:
\end{tabular}

Table 2: Modified ultra-rush VIT and maintenance therapy.

\begin{tabular}{|l|l|l|l|}
\hline No. of patients & Total 90 & Bee venom & Wasp venom \\
\hline Minimum & 4 & & \\
Maximum & 17 & & \\
Age (years, mean) & 9.3 & $18(\%)$ & $38(\%)$ \\
\hline $\begin{array}{l}\text { Sex (n0.) } \\
\text { Male }\end{array}$ & $56(\%)$ & $20(\%)$ & $14(\%)$ \\
Female & $34(\%)$ & & \\
\hline Prior sting reaction & & & \\
Grade I & $7(7.8 \%)$ & \\
Grade II & $17(18.9 \%)$ & & \\
Grade III & $58(64.4 \%)$ & & \\
Grade IV & $8(8.9 \%)$ & & \\
\hline
\end{tabular}

Table 3: Clinical data of children.

sera using the Phadia-ImmunoCAP System (Thermo Fisher Scientific). Data $>0.35 \mathrm{kU} / \mathrm{l}$ were considered positive.

Ninety patients ( 56 boys and 34 girls) aged 4 to 17 years (mean age, 9.3 years) have been given VIT thus far in accordance with a modified ultra-rush protocol consisting of just eight injections. The interval between the doses was 30 minutes. Patients did not receive pretreatment with antihistamines. The final dose of $100 \mu \mathrm{g}$ was administered on the morning of the second day of hospitalization. The cumulative venom dose was $251.11 \mu \mathrm{g}$. Therapy consolidation was achieved with $100-$ $\mu \mathrm{g}$ boost injections after 7 and 21 days; maintenance therapy was administered every 4-6 weeks (Table 2).

\section{Results}

Ninety patients received ultra-rush VIT; in all 720 injections were administered. Patient characteristics are described in table 3. All patients experienced local reactions under VIT. Of these, 20 patients had erythema $(>5 \mathrm{~cm}-20 \mathrm{~cm}$ ), and 15 patients developed significant wheals $(5 \mathrm{~cm}-15 \mathrm{~cm})$. The majority of local reactions occurred after a single dose of 40-80 $\mu \mathrm{g}$ and a median cumulative dose of $151.1 \mu \mathrm{g}$. All but one reaction occurred within 30 minutes after injection of venom. Systemic reactions were observed during preliminary treatment in two patients. One patient developed mild dyspnea after injection of 100 $\mu \mathrm{g}$, which was treated with $100 \mathrm{mg}$ of i.v. prednisolone and inhaled salbutamol. One girl had a systemic late reaction (urticaria) one hour after the last administration of $100 \mu \mathrm{g}$, which could be controlled with intravenous antihistamines. Premature termination of treatment was not required in any patient. Both of the above mentioned patients had a bee venom allergy. There was no difference in the local or systemic reactions with regards the VIT regime (ALK SQ vs. Venomil).

With regard to local reactions, no differences were observed between patients with a bee or a wasp venom allergy. Maintenance therapy has been tolerated well by all patients thus far.

\section{Discussion}

The indication for VIT is usually established with great caution in children and adolescents aged $<16$ years with sting reactions limited to the skin. It is assumed that subsequent stings will not cause general reactions in the majority of patients, and that such symptoms, if they do arise, will be limited to the skin [8].

Various dose titration protocols have been suggested for the initiation of VIT $[9,10]$. The aim is to achieve rapid protection, especially during the flight time of the insects [11]. The ultra-rush procedure is the briefest one for initiation of VIT and was first described by Zwan et al. [12]. It has been used frequently in the last few years, especially in adult patients with insect venom allergy $[2,3,13,14]$. However, the safety and efficacy of this treatment is controversially discussed. Data concerning its use in children are very scarce.

Adverse systemic reactions under ultra-rush immunotherapy for more than 210 minutes were described by Birnbaum et al. [3]. The reactions were mainly those on the skin, such as urticaria, and/or angioedema. According to Müller, grade systemic reactions of severity grades III and IV were very rare. A recent study in 94 children who were given a cumulative dose of $111.1 \mu \mathrm{g}$ Hymenoptera venom over 210 min yielded similar results [15].

Brehler et al. compared the safety of different immunotherapy protocols and noted a significant reduction in the incidence of adverse effects when fewer subcutaneous injections were administered in the preliminary phase [2]. A number of investigations concerning the safety and tolerability of various immunotherapy protocols demonstrated results similar to those reported thus far $[12,16]$.

The modified ultra-rush procedure we used proved reliable in children and adolescents with an insect venom allergy in terms of efficacy as well as tolerability. The ultra-rush procedure is also advantageous for children and parents in terms of the time involved, and the achievement of more rapid protection from repeated stings.

The maximum dose of $100 \mu \mathrm{g}$ was achieved after just 24 hours. Patients developed a serious systemic reaction after 720 injections. However, one may anticipate marked local reactions.

All patients could be discharged from the hospital at the latest after 48 hours. Compared to adults, children appear to rarely experience a systemic reaction during the titration phase of venom immunotherapy. In our investigation only two patients with a bee venom allergy had mild systemic reactions. Adult patients with a wasp venom allergy appear to tolerate the short treatment regimen better than those with a bee venom allergy $[2,3]$. In general, side effect rates following specific immunotherapy are higher for bee venom than wasp venom. Side effect rates may be as high as $40 \%$ [15].

However, a conclusive evaluation cannot be made yet because just a few systematic studies performed thus far in children permit a reliable comparison of different protocols in respect of their efficacy and tolerability.

With due awareness of the fact that a child may demonstrate a serious reaction to an insect venom injection in rare cases, VIT should be initiated under constant observation and adequate emergency treatment should be available at all times. Even when treatment is continued on an out-patient basis, adequate emergency therapy should 
Citation: Steiß JO, Lindemann H, Zimmer KP (2013) Safety of Modified Ultra-Rush Venom Immunotherapy in Children. J Aller Ther 4: 134. doi:10.4172/2155-6121.1000134

Page 3 of 3

be available at all times. The risks of insect venom allergy are still underestimated. Optimal care consists of an integral treatment concept based on acute therapy, emergency measures, diagnostic procedures for allergy, and VIT.

\section{Conflict of Interest}

J.O.Steiß received reimbursement of costs for his participation in congresses and remunerations for his lectures at scientific conferences from Bencard Company, Munich. The remaining authors have no conflicts to declare pertaining to this article

\section{References}

1. Przybilla B, Ruëff $F(2012)$ Insect stings: clinical features and management. Dtsch Arztebl Int 109: 238-248.

2. Brehler R, Wolf H, Kütting B, Schnitker J, Luger T (2000) Safety of a two-day ultrarush insect venom immunotherapy protocol in comparison with protocols of longer duration and involving a larger number of injections. J Allergy Clin Immunol 105: 1231-1235

3. Birnbaum J, Ramadour M, Magnan A, Vervloet D (2003) Hymenoptera ultrarush venom immunotherapy (210 min): a safety study and risk factors. Clin Exp Allergy 33: 58-64.

4. Roll A, Hofbauer G, Ballmer-Weber BK, Schmid-Grendelmeier P (2006) Safety of specific immunotherapy using a four-hour ultra-rush induction scheme in bee and wasp allergy. J Investig Allergol Clin Immunol 16: 79-85.

5. Konstantinou GN, Manoussakis E, Douladiris N, Hatziioannou A, Giavi S, et al. (2011) A 5-year venom immunotherapy protocol with $5011 / 4 \mathrm{~g}$ maintenance dose: safety and efficacy in school children. Pediatr Allergy Immunol 22: 393397.

6. Bonifazi F, Jutel M, Biló BM, Birnbaum J, Muller U; EAACI Interest Group on
Insect Venom Hypersensitivity (2005) Prevention and treatment of hymenoptera venom allergy: guidelines for clinical practice. Allergy 60: 1459-1470.

7. Mueller HL (1966) Diagnosis and treatment of insect sensitivity. J Asthma Res 3: $331-333$.

8. Valentine MD, Schuberth KC, Kagey-Sobotka A, Graft DF, Kwiterovich KA, et al. (1990) The value of immunotherapy with venom in children with allergy to insect stings. N Engl J Med 323: 1601-1603.

9. Finegold I (2008) Issues in stinging insect allergy immunotherapy: a review. Curr Opin Allergy Clin Immunol 8: 343-347.

10. Goldberg A, Yogev A, Confino-Cohen R (2011) Three days rush venom immunotherapy in bee allergy: safe, inexpensive and instantaneously effective. Int Arch Allergy Immunol 156: 90-98.

11. Golden DB, Kelly D, Hamilton RG, Craig TJ (2009) Venom immunotherapy reduces large local reactions to insect stings. J Allergy Clin Immunol 123: 13711375 .

12. van der Zwan JC, Flinterman J, Jankowski IG, Kerckhaert JA (1983) Hyposensitisation to wasp venom in six hours. Br Med J (Clin Res Ed) 287: 1329-1331.

13. Mamessier E, Birnbaum J, Dupuy P, Vervloet D, Magnan A (2006) Ultra-rush venom immunotherapy induces differential $T$ cell activation and regulatory patterns according to the severity of allergy. Clin Exp Allergy 36: 704-713.

14. Sturm G, Kränke B, Rudolph C, Aberer W (2002) Rush Hymenoptera venom immunotherapy: a safe and practical protocol for high-risk patients. J Allergy Clin Immunol 110: 928-933.

15. Köhli-Wiesner A, Stahlberger L, Bieli C, Stricker T, Lauener R (2012) Induction of specific immunotherapy with hymenoptera venoms using ultrarush regimen in children: safety and tolerance. J Allergy (Cairo) 2012: 790910.

16. Laurent J, Smiejan JM, Bloch-Morot E, Herman D (1997) Safety of Hymenoptera venom rush immunotherapy. Allergy 52: 94-96. 